Emmanuel Oyekanlu

eo48@drexel.edu

Drexel University, Philadelphia, Pennsylvania, USA

\title{
Fuzzy Inference Based Stability Optimization for loT Data Centers DC Microgrids: Impact of Constant Power Loads on Smart Grid Communication over the Powerline
}

\section{SUMMARY}

Direct Current (dc) microgrids due to their efficiency and energy savings are being deployed to provide power for servers in Internet of Things (loT) data centers, in more electric aircrafts (MEA), electric ships and in rail systems round the word. In this paper, Takagi-Sugeno fuzzy inference method is used to establish a Lyapunov stability candidate for a $380 \mathrm{~V}$ ring bus de microgrid modeled with Matlab. To determine suitability of using powerline communication (PLC) to monitor stability condition on the $380 \mathrm{~V} \mathrm{dc}$ microgrid, impact of distortion caused by microgrid constant power loads (CPL) on signals transmitted over the dc microgrid PLC channel is examined. It is shown in this paper that while Lyapunov asymptotic stability is maintained on the dc bus, increasing CPL on the microgrid causes the dc microgrid PLC channel to experience growing signal distortion.

\section{KEYWORDS}

microgrid, Lyapunov, fuzzy inference, stability, powerline communication,

\section{MOTIVATION AND INCITEMENT}

Worldwide, data centers (such as Google Data Center) consume up to $1.5 \%$ of total generated electricity round the world. From the consumed $1.5 \%$, they utilize only about $30 \%$ leading to a loss of $70 \%$. Thus, the efficiency ratio of data centers round the world is approximately $30 \%$ [1]. The reason for this low efficiency is that data centers are always over-engineered in terms of electricity provision and usage with a lot of redundancies in electric power provision introduced in data center networks. An average data center draws about $25 \mathrm{MW}$ of electricity from the grid, hence they present a huge load to the electricity grid. Data centers must be reliable, they must have the exact needed supply of electricity at the right time since most data center equipment are very sensitive. Consider the typical data center power distribution topology shown in Figure 1. AC supply from the electricity mains will be 


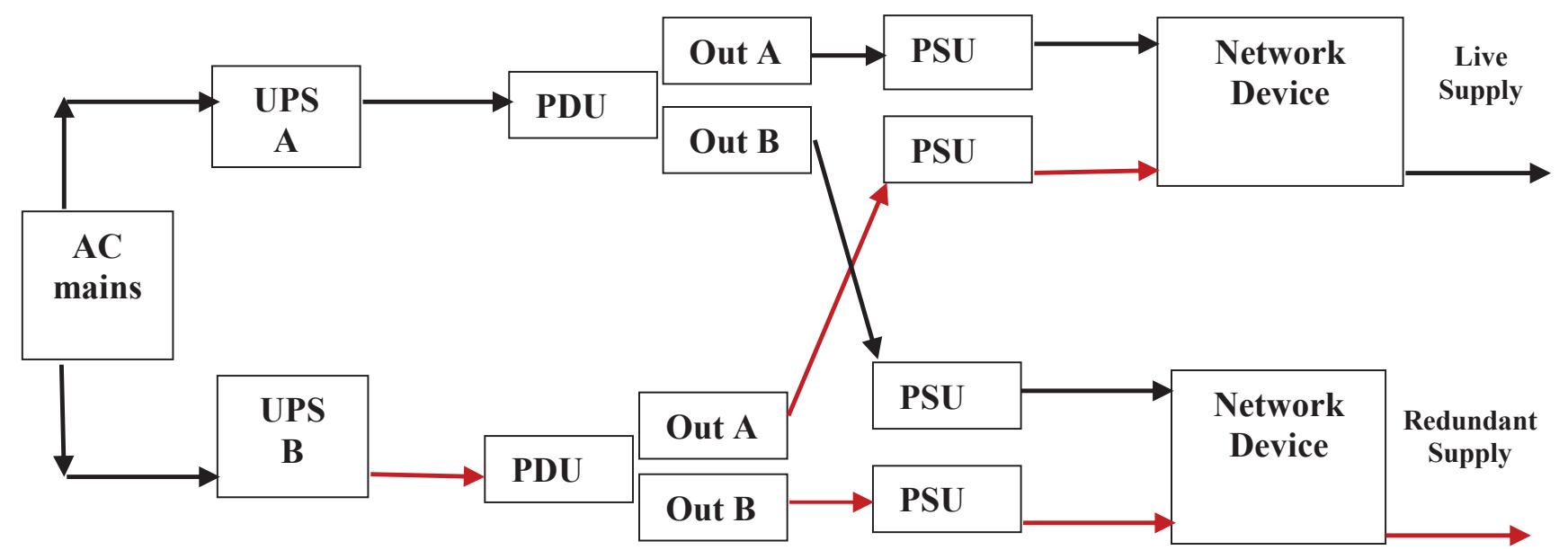

Figure 1. Data centers looses enormous amount of power due to overprovisioning of redundancies.

supplied to the Uninterruptible Power Supply (UPS), and from the UPS to the Power Distribution Unit (PDU). It will be converted to dc power at the Power Supply Unit (PSU) and finally to network servers, switches, huge database computers, and other general network devices. The auxilliary part of the topology (denoted with red arrows) that result to redundant power supply is only introduced to provide added reliability to the network. In most cases, the reducndant power supply is not used, leading to losses of generated electricity. To curtail these enormous losses and to ensure that microgrid stability is maintained, it is envisaged that by installing a communication overlay such as PLC with the power distribution topology, then downstream connected devices will be visible to upstream UPS by means of communication and the UPS can adjust power needs based on the number of connected devices [2]. With PLC, downstream visibility of connected devices will be achieved, thus power consumption efficiency and load balancing can be improved, and the need for redundant power supplies will be progressively phased out in data center topologies. Data center power usage and stability monitoring using PLC has been suggested in [2], but no one have work on its feasibility and ways to make this possible. Also, to reduce the incidence of power losses, and to improve on reliability and efficiency, ring bus dc microgrids are being increasingly used in data centers, MEA, electric ships, rail systems, and autonomous islands more than ever [3]. DC microgrids have significant advantages when compared to alternating current (AC) based microgrids. dc microgrid have fewer conversion stages, leading to lower energy losses. Also. Most renewable energy sources such as photovoltaic (PC) are dc in nature. In fact, dc microgrids are central to the development of smart grid systems [3]. However, for PLC to be a reliable means of monitoring loT data center stability and power usage, the impact of varying DC microgrid powerline channel power loads on the effectiveness, signal distortion and reliability of PLC must be further studied. This is the main contribution of this paper.

\section{LITERATURE REVIEW}

In [4], authors discussed a power quality $(\mathrm{PQ})$ monitoring solution based on PLC. The PLC hardware and software used for PQ monitoring and evaluation is based on the IEEE std. 1159. It is shown that PLC could be applied for power management by the end user. However the effect of PLC channel loads on the PQ monitoring effectiveness of the system designed is not discussed. In [5], changes in key PLC channel characteristics of the channel frequency response (CFR) is used to observe the PQ of the powerline. In [6], PLC is used as a communication link between a smart meter and a central server. The central server can be used to monitor the power usage of a smart meter. In [7], PLC is used for monitoring and supervision of feeder equipment for medium voltage substation automation. In [8], PLC is used for monitoring Electrocardiogram (ECG) and Electroencephalogram (EEG) signals in order to reduce the cost associated with big health data transmission in healthcare platforms. However, in all of the research works considered, the impact of varying PLC channel loads on the effectiveness of PLC is not examined.

\section{CONTRIBUTION AND ORGANIZATION}

In this paper, we have examined the stability of a low voltage dc microgrid that could satisfy the energy needs of a small autonomous system such as aircrafts, loT data centers and electric ships when channel loads are varying on the powerline channel of the dc microgrid. A $380 \mathrm{~V}$ dc bus ring is modeled using Matlab due to many research work that reports on the merits of deploying $380 \mathrm{~V}$ dc for data centers and autonomous power systems worldwide [1] - [3], [9], [10]. Small and large signal stability issues are examined for the modeled $380 \mathrm{~V}$ dc microgrids when loT cloud servers or data center servers acting as CPL are increasing on the dc bus. Stability is also examined when an energy source is experiencing large signal voltage transients on the microgrid. Impact of CPL loads on distortion experienced by basedband signals sent over the dc bus when the dc bus is used as a communication channel is also examined. Special emphasis is placed on dc microgrids that are providing power for loT cloud servers and data centers in this paper due to the energy demands of such data centers and the impact of the demands on power systems that support them worldwide. Our contribution in the paper, to the best of our knowledge is the first known attempt at understanding the relationship between dc microgrid stability, varying CPLs and amount of signal distortion that they can cause of PLC signals transmitted on dc microgrid channels.

Section two of this paper discussed problem of circulating current, electromagnetic interference (EMI) and dc bus line impedance problem on the $380 \mathrm{~V}$ dc ring bus microgrids. Small signal stability issues using Bode plots are discussed extensively. In section three, modeling approach for the 380 $\mathrm{V}$ dc ring bus microgrid used in this paper is discussed. In section four, the Takagi-Sugeno (TS) fuzzy inference method used to establish a Lyapunov candidate to ensure stability of the microgrid is discussed. Stability of the $380 \mathrm{~V}$ dc ring bus microgrid is discussed as well. In section five, impact of CPL on distortion of signal communicated using PLC on the dc bus of the microgrid is examined. Section six is conclusion of the paper.

\section{CIRCULATING CURRENT IN LVDC RING BUS MICROGRID}

A representation of the ring bus LVDC microgrid considered in the work is shown in Figure 2. In this configuration, there exist several upstream lineregulating converters (LIR) that are connected to different energy sources which in most cases are renewable energy sources. LIR converters could be ac-dc or dc-dc, but the LIR converter discussed in this paper are Buck dc-dc converters. There also exist several point of load (POL) converters that are connected to loads. The LIR converters considered are Buck converters and they are stepping down a high voltage $600 \mathrm{~V}$ (considered in this paper) to a lower voltage, $380 \mathrm{~V}$. On the modeled dc microgrid, only dc-dc Buck converters are considered for both LIR and POL converters. Similar work that considered ring bus Microgrids are reported in [10] and [11]; however, both works considered LIR and POL buck converters that have similar parameters. This is not quite close to real life since in most cases, Microgrids are populated with converters that that have quite different configurations and parameters. 


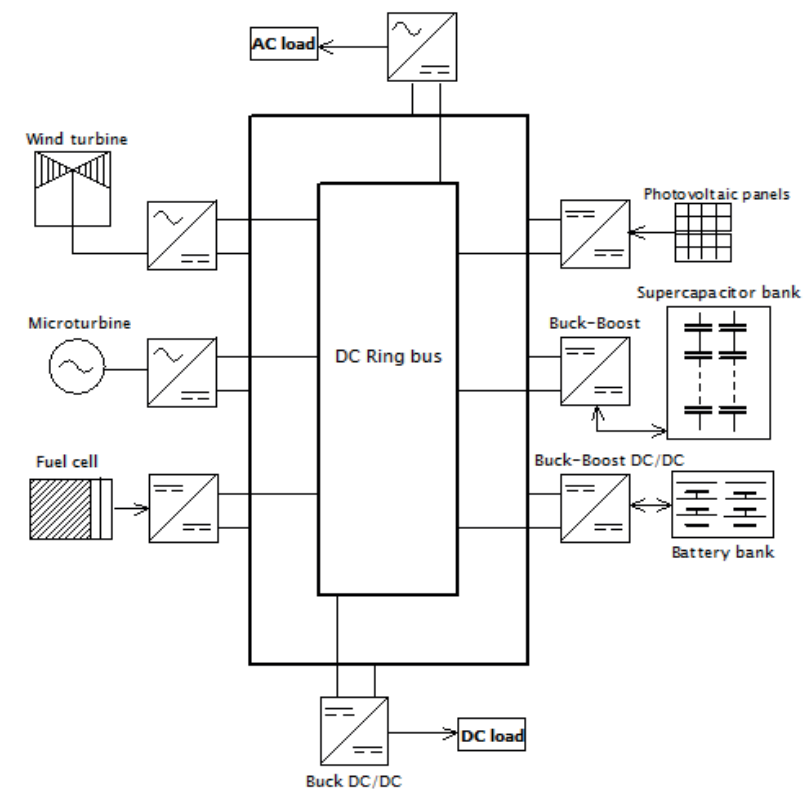

Figure 2. Low Voltage LVDC ring bus model

In addition, most work in literature always consider constant power high bandwidth loads (CPL) as the worst-case load that could jeopardize stability and make control effort in Microgrids to be difficult. However, report in [12] and [13] have reveal new set of complex impedance low bandwidth loads that could exist because of changing converter parameters and other systemic nonlinearities that can exist on the microgrid in any instance. In addition to stability challenges that can result from changing converters bandwidth, problem of circulating currents can also jeopardize stability and power quality on dc microgrids. Power quality issues are very important for loT cloud server, aircrafts and electric ship loads using dc microgrids. When dc-dc converters are connected on microgrids for load sharing, problem of circulating current always exist. It is often a direct result of poorly regulated dc voltage and complex impedance that exist on the dc bus. For a multi-converter microgrid, the flow of circulating current among converters is as shown in Figure 3.

Circulating current can exist between converter 1, 2 and 3. Converter 1 (Conv 1 in Figure 3 ) and 2 are LIRs while converter 3 is a POL converter. Indeed, circulating current flow (indicated by arrows and dotted lines) could exist among all the converters on the microgrid as shown in Figure 3.

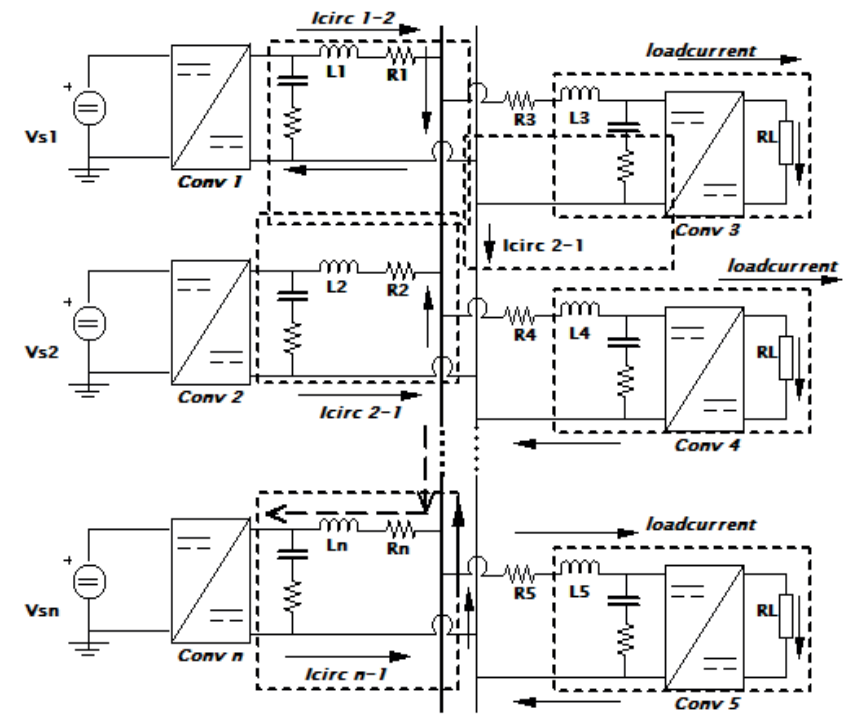

Figure 3. Circulating current problem among a multi-converter system

As reported in [14], Kirchhoff voltage law (KVL) and Kirchhoff current law $(\mathrm{KCL})$ can be used to analyze the amount of circulating currents that can exist on the microgrid. Using KVL, an assessment of converter output currents between converter 1 and 2 when complex line impedance is considered can be given as:

$$
\begin{aligned}
& I_{1}=\frac{\left(Z_{2}+Z_{L}\right) V_{S 1}-Z_{L} V_{S 2}}{Z_{1} Z_{2}+Z_{1} Z_{L}+Z_{2} Z_{L}} \\
& I_{2}=\frac{\left(Z_{1}+Z_{L}\right) V_{S 2}-Z_{L} V_{S 1}}{Z_{1} Z_{2}+Z_{1} Z_{L}+Z_{2} Z_{L}}
\end{aligned}
$$

Source voltages can be expressed as

$$
\begin{aligned}
& V_{s 1}=I_{1} Z_{1}+I_{L} Z_{L} \\
& V_{s 2}=I_{2} Z_{2}+I_{L} Z_{L}
\end{aligned}
$$

and the difference between (3) and (4) is

$$
V_{s 1}-V_{s 2}=I_{1} Z_{1}-I_{2} Z_{2}
$$

The circulating currents between converter 1 and 2 can be expressed as when $Z_{1} \neq Z_{2}$

$I_{\operatorname{circ1} 1}=-I_{\operatorname{circ} 2}=\frac{V_{S 1}-V_{S 2}}{Z_{1}+Z_{2}}=\frac{I_{1} Z_{1}-I_{2} Z_{2}}{Z_{1}+Z_{2}}$

Also, when $\mathrm{Z}_{1}=\mathrm{Z}_{2}$

$$
I_{\operatorname{circ} 1}=-I_{\operatorname{circ} 2}=\frac{\left(I_{1}-I_{2}\right) Z_{1}}{2 Z_{1}}=\frac{\left(I_{1}-I_{2}\right)}{2}
$$

The circulating current for the $\mathrm{n}$ parallel converters from converter 1 to all other converters when (when is given by

$$
\begin{aligned}
I_{\operatorname{circ1} 1} & =\frac{I_{1} Z_{1}-I_{2} Z_{2}}{Z_{1}+Z_{2}}+\frac{I_{1} Z_{1}-I_{3} Z_{3}}{Z_{1}+Z_{3}}+\cdots+\frac{I_{1} Z_{1}-I_{n} Z_{n}}{Z_{1}+Z_{n}} \\
I_{\operatorname{circ1}} & =\sum_{k=2}^{n} \frac{I_{1} Z_{1}-I_{k} Z_{k}}{Z_{1}+Z_{n}}
\end{aligned}
$$

also, the circulating current for $\mathrm{n}$ parallel converters from converter 1 to all other converters when is given by

$$
\begin{aligned}
& I_{\operatorname{circ1} 1}=\frac{I_{1}-I_{2}}{n}+\frac{I_{1}-I_{3}}{n}+\cdots+\frac{I_{1}-I_{n}}{n} \\
& I_{\operatorname{circ1}}=\sum_{k=2}^{n} \frac{I_{1}-I_{k}}{n}
\end{aligned}
$$

Both results above in (8) and (11) could be [14] extended to the case for converters $2,3, \ldots ., n$, for I $I_{\text {circ n }}$. 


\section{EMI and Line Impedance Problems on DC Microgrids}

Apart from circulating currents, other issues that can affect stability is the existence of line electromagnetic interference (EMI) and excessive line impedance, both of which if not confined within tolerable limits can jeopardize individual converter's small signal stability and hence the entire microgrid large signal stability. To limit EMI and input impedance to individual converters, damped input filters are always used. There are several possible input filter configurations to choose from [15], and the configurations are mainly based on the mode of damping employed to reduce the filters output impedance. Filter output impedance are a major consideration if both small and large signal stability of the microgrid are to be preserved. Middlebrook criterion [15] states that for stability to be maintained, then the magnitude of filter's output impedance, i.e., ZoutputFilter in Figure 4(a) must be less than the magnitude of the converter's input impedance ZinputConverter; i.e.,

| ZoutputFilter ||$<<||$ ZinputConverter

For Middlebrooks criterion to be met, then filters must be damped. Reason is that, for undamped input filter in Figure $4 \mathrm{a}$, at resonant frequency f0, that is, when:

$$
f_{o}=\frac{1}{2 \pi \sqrt{L C}}
$$

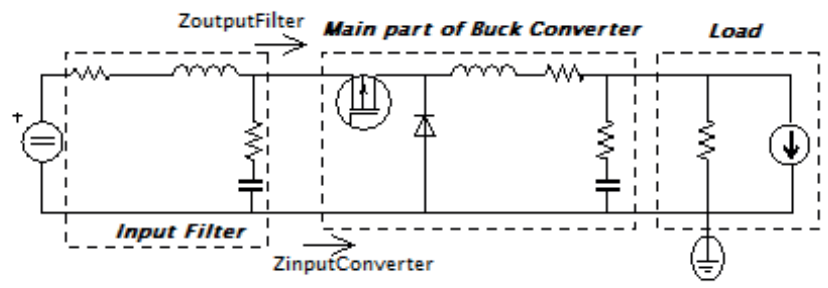

Figure 4a. Undamped filter with Buck converter.

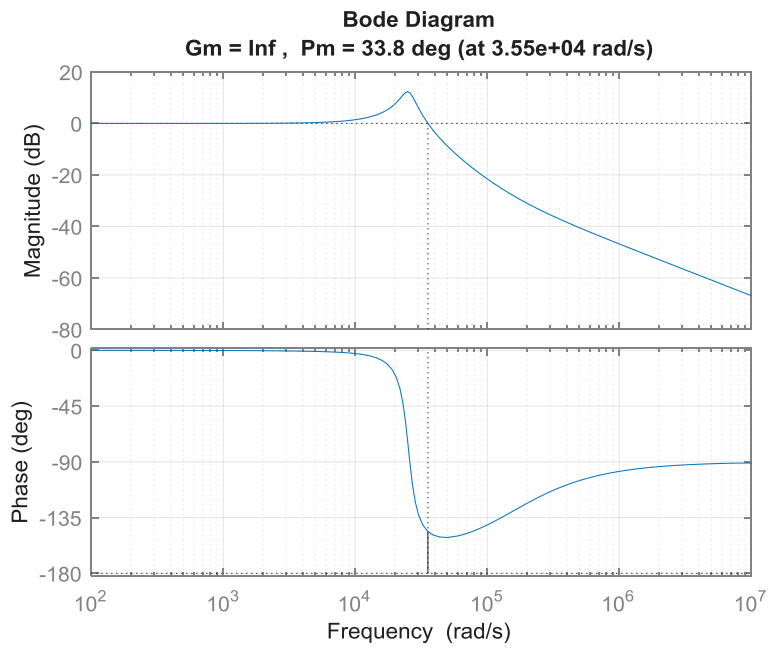

Figure 4b. Small Signal Bode plot for undamped input filter.
ZoutputFilter will approach infinity [15]. Since this work focuses on stability issues and distortion suffered by signals transmitted on dc microgrid channels, then it is instructive to examine different input filter configurations and how those configurations impact small signal converter stability and overall large signal system-wide stability. Major existing input filter topologies [15], [16], [17], that are examined include undamped input filter, parallel damped input filter (Figure 5a), series damped input filter (Figure 6a).

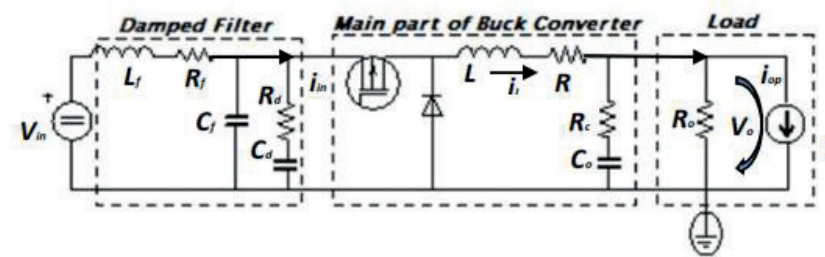

Figure 5a. Parallel damped filter

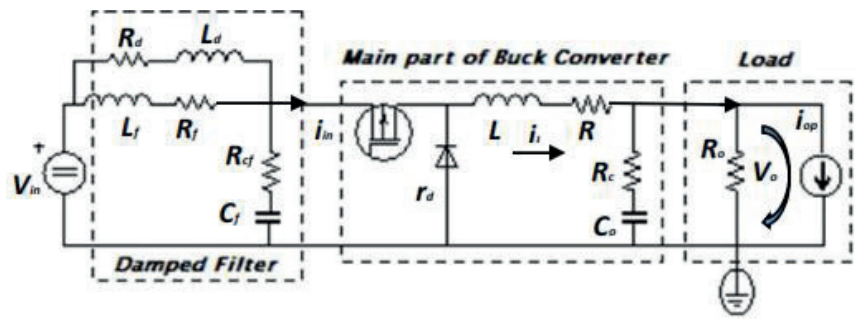

Figure 6a. Series damped filter

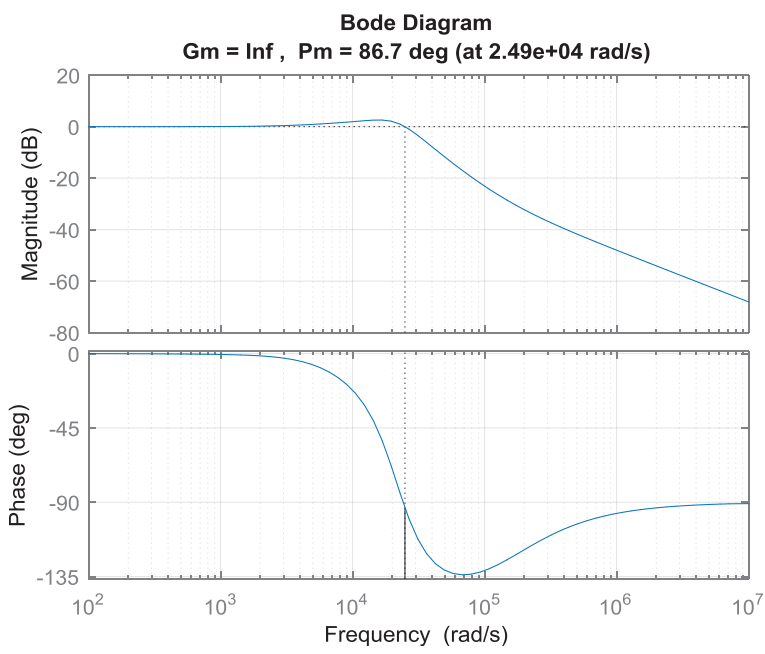

Figure 5b. Small Signal Bode plot for parallel damped input filter.

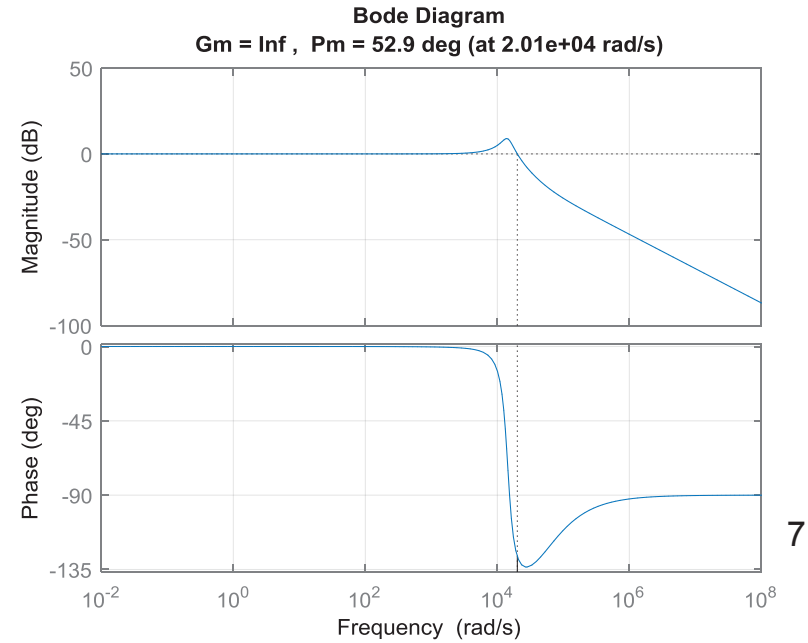

Figure 6b. Small Signal Bode plot for series damped input filter. 
For each configuration, bode plots are generated. The phase margin of bode plots is an indication of the limit of phase variation that can be attained at crossover frequency before dc microgrid stability is lost. The gain margin also indicates the limit of gain variation attainable at crossover frequency before stability is jeopardized. For most systems, a phase margin of 60-degree or more is always desired. Thus, as shown in Figure 4b, undamped input filter will adversely affect microgrid stability because of its poor phase margin of 33 degree. Stability result for a parallel damped configuration is shown in Figure $5 \mathrm{~b}$ where it is show that the phase margin is well above the 60-degree threshold. The phase margin of the series damped filter of Figure $6 \mathrm{~b}$ is lower than the 60 -degree threshold. This indicate that the series damped filter may not yield a good stability performance if it is used on the microgrid.

\section{RING BUS MICROGRID MODELING}

The ring bus microgrid considered in this paper is modeled as a contiguous series of pi bus (Figure 7) sections [10], [18]. Based on the segmenting approach discussed in [18], a breakpoint is introduced on the microgrid bus as shown in Figure 8. Each pi segment is also modeled as a filter and is added with the EMI-limiting

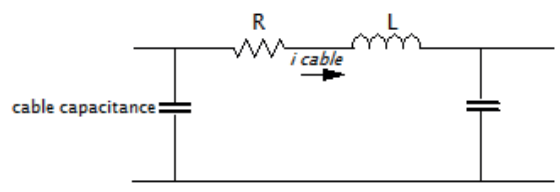

Figure 7. Pi section model of transmission line used to segment the dc bus in Figure

damped input filter already existing with each converter as shown in Figure 9 a. The result is a multiple section input filter with a good 180-degree phase margin bode plots shown in Figure 9b.
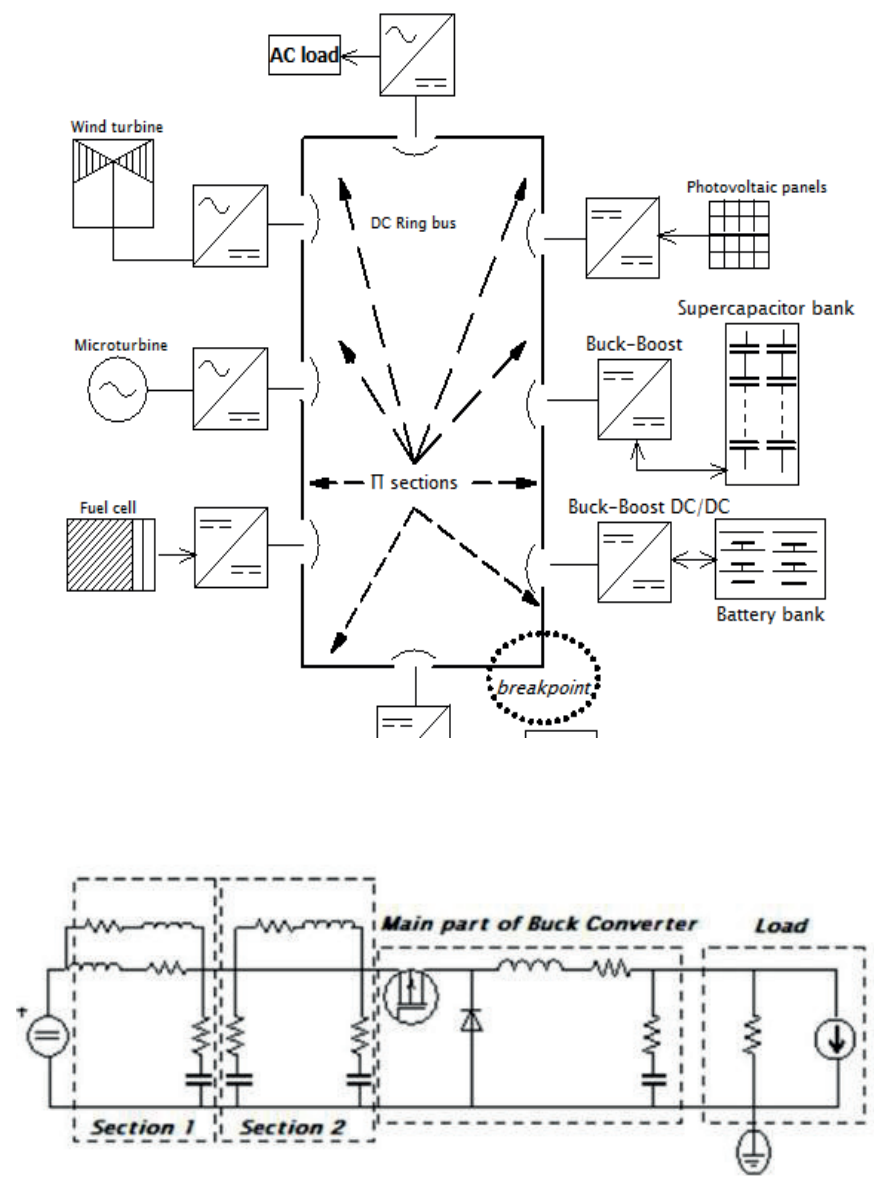

Figure 9a. Multiple section input filter representing EMI filter and pi section lines

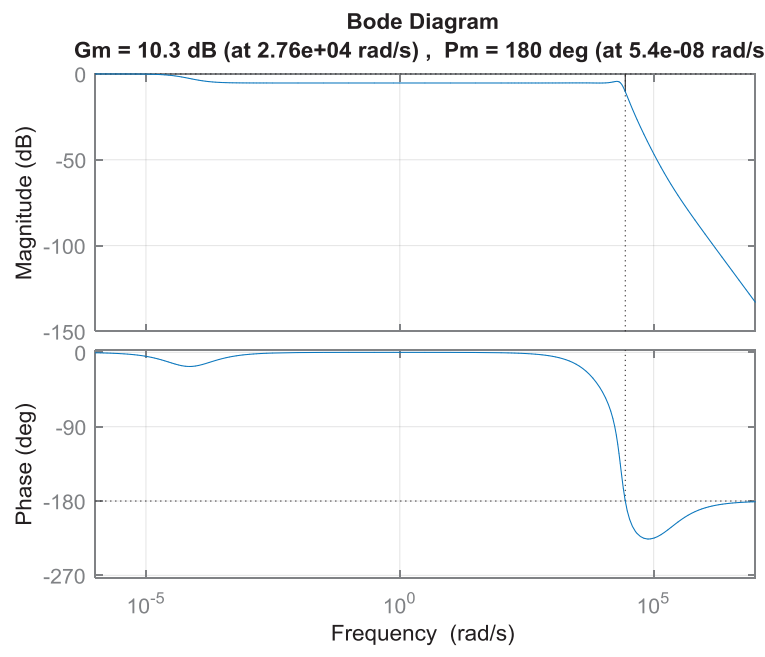

Figure 9b. Bode plot of the resulting multiple section input filter

\section{FUZZY INFERENCE DC MICROGRID LYAPUNOV LARGE SIGNAL STABILITY REGION ESTIMATION USING TAKAGI- SUGENO INFERENCE METHOD}

To estimate the stability region and the impact of CPL distortion on signals exchanged over the dc bus of a microgrid having CPL, a pi section of the dc ring bus microgrid having one-source and one CPL shown in Figure 11 is used. This is similar to the approach used in [21], [22], [23], [25]. The generalized load impedance model on dc microgrids given in [12], [13], [20] is

$$
Z_{L}(s)=\frac{\sum_{i=o}^{m} b_{i} s^{i}}{\sum_{i=o}^{n} a_{i} s^{i}} \quad b_{i} a_{i} \varepsilon \mathrm{R}
$$

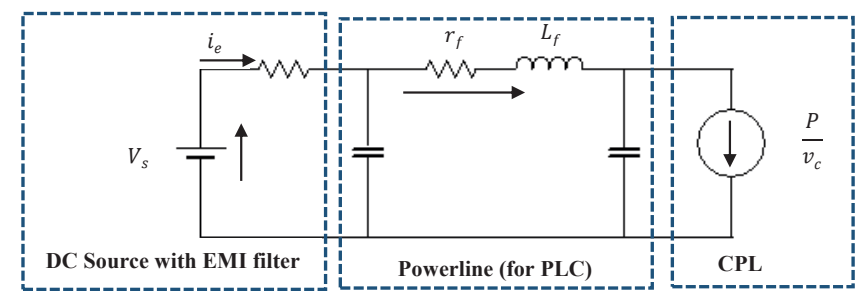

Figure 11. Model of a dc microgrid with one source, PLC and a CPL (one-load) used in this study

The constants $\mathrm{m}$ and $\mathrm{n}$ in (14) are the numerator and denominator orders, and and are load coefficients. For downstream close-loop POL converters, the input impedance in view of load impedance of (14) will produce:

$$
Z_{i}(s)=\frac{\sum_{i=o}^{m} b_{i}\left[n_{3} s^{i+3}+n_{1} s^{i+1}+n_{0}\right]+\sum_{i=o}^{n} a_{i} n_{2} s^{i+2}}{\sum_{i=o}^{m} b_{i} d_{2} s^{i+2}+\sum_{i=o}^{n} a_{1}\left[d_{1} s^{i+1} d_{0}\right]}
$$

The load model can be used to understand the nature of different loads that could exist on the DC Microgrid. The generalized load model (15), could be used to represent CPLs, constant impedance loads, first order lag loads and other load types that could incidentally exist because of faults on the microgrid or the changing nature of the dc converters having varying parameters. 


\section{Takagi-Sugeno Fuzzy Inference Method}

The Takagi-Sugeno (TS) fuzzy inference model is a very good universal approximator for nonlinear systems [19] such as a one-source and a CPL load model of the dc microgrid of Figure 11. In TS fuzzy inference models, a nonlinear system could be decomposed into a subset of linear subsystems of the type $=\mathrm{x}^{\prime}(\mathrm{t})=A_{t} \mathrm{x}^{\prime}(\mathrm{t})+B_{i} u(t)$. These linear models whose local behavior is defined by the $i^{\text {th }}$ fuzzy rule are interconnected by nonlinear scalar functions, and are a convex sum of the original nonlinear system [21]. Lyapunov theorem can then be applied to establish stability or other wise of the TS fuzzy inference model.

For each susbystem $\dot{x}(\mathrm{t})$ of the whole model, a premise for $i=1,2, \ldots ., q$; where $q$ is the number of nonlinearity (for example, operating point) is established. Each subsystem is further linked to a weigthed activation function that will determine the membership degree of the linear subsystem in the whole fuzzy model of the nonlinear system. The decision space of the whole nonlinear system is partitioned into a fuzzy inference rule based space of the form: for rule $\mathrm{R}^{\mathrm{i}}$ : if $\mathrm{z}_{1}(\mathrm{t})$ is activated by $\mathrm{F}_{\mathrm{i}}^{1}, \ldots \ldots$. , and $\mathrm{z}_{\mathrm{q}}(\mathrm{t})$ is activated by $\mathrm{F}_{\mathrm{i}}^{1}$, then the system can be modeled by

$$
\left\{\begin{array}{l}
\dot{x}(t)=A_{i} x(t)+B_{i} u(t) \\
y(t)=C_{i} x(t)
\end{array}\right.
$$

In (16), , , and are constant matrices. The weight attributed to the rule of each subsystem of the linear fuzzy model is given as

$$
\omega_{i}=\omega_{i}(z(t))=\prod_{j=1}^{q} F_{j}^{i}\left(z_{j}(t)\right), \quad i=1,2, \ldots,
$$

and the weights given in (17) can be normalized using

$$
h_{i}(z(t))=\frac{\omega_{i}(z(t))}{\sum_{i}^{r} \omega_{i}(z(t))}, i=1,2, \ldots \ldots, r
$$

which verifies the property convex sum

$$
\sum_{i}^{r} h_{i}(z(t))=1, h_{i}(z(t)) \geq 0 \forall t
$$

For the whole nonlinear model, the fuzzy inference model for the whole nonlinear dc microgrid system can be represented as

$$
\left\{\begin{array}{l}
\dot{x}(t)=\sum_{i}^{r} h_{i}(z(t)) A_{t} x(t)+B_{i} u(t) \\
y(t)=\sum_{i}^{r} h_{i}(z(t)) C_{i} x(t)
\end{array}\right.
$$

The TS theorem leads to a generation of the Lyapunov function $V(x)$ that allows the determination of the domain of attraction for a given operation point. The existence of a Lyapunov candidate based on the TS model shows that the given operation point is asymptotically stable. The resulting fuzzy inference model showing this asymptotic stability can then be represented as a system of linear matrix inequality (LMI) which is an optimal convex solution of the Lyapunov stability criteria. The feasibility of the LMI of the converter is established by solving LMI equations in (21) to obtain a matrix $\mathrm{M}$ that is positive definite according to Lyapunov criteria.

$$
\left\{\begin{array}{c}
M=M^{T}>0 \\
A_{1}^{T} \cdot M+M \cdot A_{1}<0 \\
A_{2}^{T} M+M \cdot A_{2}<0, \ldots \ldots \ldots \ldots, A_{i}^{T} M+M \cdot A_{i}<0, \text { for } \forall_{i} \in\{2, \ldots \ldots, r\}
\end{array}\right.
$$

Existence of a positive definite $M$ establishes the feasibility of the fuzzy models generated. A large signal Lyapunov candidate solution can then be constructed based on the M matrix generated [19], [23]. The large signal domain that shows the domain of stability of the microgrid based on the rated power and the load type is then determined. The existence of $M$ is based on satisfying two conditions. First, all local linear subsystem must be stable. For this to be possible, the matrices in (21) must be Hurwitz. Secondly, if $A=\sum_{i=1} A_{i}$ is Hurwitz, then a Lyapunov function, which proves the stability of the linear local model must also exist.

For the dc microgrid model shown in Figure 11 with $\mathrm{CPL}\left(p / v_{c}\right)$, a suitable operating point for the CPL defined by inductor current and capacitor voltage with constant load power can be represented [21] as

$$
\left\{\begin{array}{l}
i_{e o}=\frac{V_{e}-v_{c o}}{r_{f}}=\frac{p_{s}}{v_{c o}} \\
v_{S o}=\frac{V_{e}+\sqrt{V_{e}^{2}-4 p_{s} r_{f}}}{2}
\end{array}\right.
$$

A change of variables $\mathrm{x}^{1}=\mathrm{i}_{\mathrm{e}}-\mathrm{i}_{\text {eo }}$ and $\mathrm{x}_{2}=\mathrm{v}_{\mathrm{s}}-\mathrm{v}_{\text {co }}$ provide avenues by which the system can be studied at its operating points. Using these change of variables, the system in Figure 11 can be linearized and studied at its operating points, leading to

$$
\left\{\begin{array}{l}
\dot{x}_{1}=\frac{-r_{f}}{L_{f}} x_{1}-\frac{1}{L_{f}} x_{2} \\
\dot{x}_{2}=\frac{1}{C} x_{1}+\frac{p_{s}}{C v_{c o}} \frac{x_{2}}{v_{c o}+x_{2}}
\end{array}\right.
$$

The system in (23) with its nonlinear part $\left(\frac{p_{S}}{C v_{c o}} \frac{x_{2}}{v_{c o}+x_{2}}\right)$ can be represented as

$$
\left[\begin{array}{l}
\dot{x}_{1} \\
\dot{x}_{2}
\end{array}\right]=\left[\begin{array}{cc}
\frac{-r_{f}}{L_{f}} & -\frac{1}{L_{f}} \\
\frac{1}{C} & f\left(x_{2}\right)
\end{array}\right]\left[\begin{array}{l}
x_{1} \\
x_{2}
\end{array}\right]=A\left(x_{2}\right)\left[\begin{array}{l}
x_{1} \\
x_{2}
\end{array}\right]
$$

In (24), $\mathrm{f}\left(\mathrm{x}_{2}\right)$ represent the system nonlinear part $\frac{p_{S}}{C v_{c o}} \frac{x_{2}}{v_{c o}+x_{2}}$. The nonlinear part can be studied within an upper and lower fuzzy inference boundary represented [21] as

$$
f\left(x_{2}\right)=f_{\max } F_{1}^{1}(z)+f_{\min } F_{1}^{2}(z)
$$

In (25), the upper and lower boundary of the dc microgrid nonlinear activation function $f\left(x_{2}\right)$ lies within the domain of the fuzzy inference rule with activation functions $\mathrm{F}_{1}{ }^{1}(\mathrm{z})$ and $\mathrm{F}_{1}{ }^{2}(\mathrm{z})$ that verifies the convexity property

$$
\left\{\begin{array}{l}
0 \leq F_{1}^{1}(z)=\frac{z-f_{\min }}{f_{\max }-f_{\min }} \leq 1 \\
0 \leq F_{1}^{2}(z)=\frac{f_{\max }-z}{f_{\max }-f_{\min }} \leq 1
\end{array}\right.
$$

$$
\left\{\begin{array}{l}
f_{\min }=\frac{p_{s}}{C v_{c o}} \frac{1}{v_{c o}+x_{2 \max }} \\
f_{\max }=\frac{p_{s}}{C v_{c o}} \frac{1}{v_{c o}+x_{2 \min }}
\end{array}\right.
$$


The two local models resulting from the single nonlinear function of the system can be represented with two rules using the activation functions $\mathrm{F}_{1}{ }_{1}$ (z) and $\mathrm{F}_{1}^{2}(\mathrm{z})$; and they are respectively represented [21] as

$$
\begin{aligned}
& {\left[\begin{array}{l}
\dot{x}_{1} \\
\dot{x}_{2}
\end{array}\right]=\left[\begin{array}{cc}
\frac{-r_{f}}{L_{f}} & -\frac{1}{L_{f}} \\
\frac{1}{C} & f \max
\end{array}\right]\left[\begin{array}{l}
x_{1} \\
x_{2}
\end{array}\right]=A_{1}\left[\begin{array}{l}
x_{1} \\
x_{2}
\end{array}\right]} \\
& {\left[\begin{array}{l}
\dot{x}_{1} \\
\dot{x}_{2}
\end{array}\right]=\left[\begin{array}{cc}
\frac{-r_{f}}{L_{f}} & -\frac{1}{L_{f}} \\
\frac{1}{C} & f m i n
\end{array}\right]\left[\begin{array}{l}
x_{1} \\
x_{2}
\end{array}\right]=A_{2}\left[\begin{array}{l}
x_{1} \\
x_{2}
\end{array}\right]}
\end{aligned}
$$

To further clarify the method of TS fuzzy inference model, value of the nonlinear part of the dc microgrid can be deduced using a TS membership function. The membership function $(M)$ of the TS fuzzy inference model can be represented as shown in Figure 12.

$$
M_{2}(z)
$$

$$
M_{1}(z)
$$

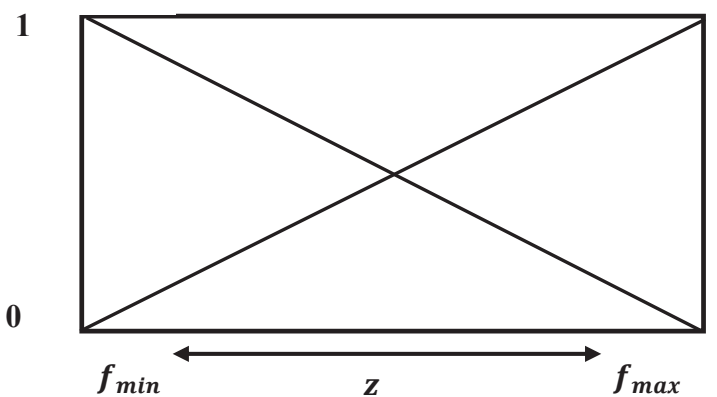

Figure 12. Takagi-Sugeno Fuzzy Inference Formulation

TS membership function can be derived such that for the nonlinear part of the dc microgrid equation in (23), membership function can be written as already discussed in (25), leading to

$$
\mathrm{Z}=f\left(x_{2}\right)=f_{\max } M_{1}(z)+f_{\min } M_{2}(z)
$$

where

$$
M_{1}(z)+M_{2}(z)=1
$$

and $F_{\text {max }}$ and $F_{\text {min }}$ are computed based on

$$
x_{2} \in\left[x_{2} \min \quad x_{2} \max \right]
$$

The fuzziness of the TS method implies that if $z$ is in the positive (+) region of the TS membership model shown in figure 12, then and the matrix of the dc microgrid can be evaluated as shown in (28). If $z$ is in the negative region of TS membership of figure 12, then dc microgrid can be evaluated as shown in (29). Using this fuzzy method, value of the nonlinear system part can be deduced, and stability of the system evaluated. The stability domain of the dc microgrid system is simulated for a data center microgrid with a $380 \mathrm{~V}$ dc bus supplying data center cloud server acting as CPLs. The data center dc bus is assumed to have a $600 \mathrm{~V}$ input voltage from renewable energy sources [3] (such as PV arrays). System parameters [3] are shown in Table 1. Using the LMI model in (21) and systems parameters in Table I, a Matlab procedure for obtaining $\mathrm{M}$ as discussed in [26] is used to obtain the needed matrix $\mathrm{M}$ for the dc microgrid system shown in Figure 11.

Table I. 600 V/ 380 V DC-DC Data Center Microgrid Study Parameters

\begin{tabular}{|l|l|}
\hline Parameter & Value \\
\hline$V_{\text {in }}$ & $600 \mathrm{~V}$ \\
\hline DC Bus Voltage $\left(V_{\text {bus }}\right)$ & $380 \mathrm{~V}$ \\
\hline Bus Capacitance $($ varied $)$ & $200 \mu \mathrm{F}, 300 \mu \mathrm{F}, 400 \mu \mathrm{F}$ and $500 \mu \mathrm{F}$ \\
\hline Data Center Server CPL (varied) & $400 \mathrm{~W}, 800 \mathrm{~W}, 1200 \mathrm{~W}$ and $1600 \mathrm{~W}$ \\
\hline
\end{tabular}

The obtained matrix $\mathrm{M}$ for a $400 \mathrm{~W}$ cloud server center CPL and its associated Lyapunov candidate [23] is

$$
\begin{aligned}
& M=\left[\begin{array}{ll}
105.3 & 0.056 \\
0.056 & 0.015
\end{array}\right] \\
& V(x)=105.3 x_{1}^{2}+0.112 x_{1} x_{2}+0.015 x_{2}^{2}
\end{aligned}
$$

The domain of attraction for the system using varying dc-bus capacitance and varying cloud server loads modeled as CPLs are shown in Figure 13 and Figure 14 respectively. Since acceptable level of dc bus capacitance for dc microgrid is still an area of research study worldwide [3], [27], [28], the impact of allowed capacitance values for the $380 \mathrm{~V} \mathrm{dc}$ microgrid on the dc bus link is shown in Figure 13. It is shown that the size of domain of attraction, and hence system stability increases with increasing dc bus capacitance. For CPLs, the system domain of attraction indicate that system stability will be jeopardized when server loads, acting as CPLs increases on the dc microgrid.

The system domain of attraction decreases when CPLs increases on the dc bus. To explicitly show this effect, the upper limit of each server load domain of attraction is traced out on the voltage axis as shown in Figure 15 , and the relationship of the domain of attraction and server loads is explicitly plotted and shown in Figure 16. The existence of a Lyapunov candidate for the modeled system ensures that the system is large-signally stable [21], [23], even when the system is experiencing large variation in input voltage. The system will maintain stability when voltage variation being experienced by the system falls within the stability margin of the domain of attraction. The asymptotic stability of the modeled $600 \mathrm{~V} / 380$ $\mathrm{V}$ dc microgrid is verified by varying the input voltages $V$ in of the system as shown in Figure 17.

In Figure 17, it is shown that when the system input voltage (blue) is varied by reducing it to $550 \mathrm{~V}$ in time 0 to $0.2 \mathrm{~s}, 0.5$ to $0.7 \mathrm{~s}, 1$ to $1.2 \mathrm{~s}$ etc., the output voltage (red), on dc bus supplied to the cloud server system is stable at $380 \mathrm{~V}$ dc. This asymptotic stability implies that the existence of Lyapunov candidate resulting from the LMI based TS fuzzy inference model makes the system to be stable in the large signal sense when the input voltage is experiencing large signal variations by decreasing in steps of $50 \mathrm{~V}$ from $600 \mathrm{~V}$ to $550 \mathrm{~V}$ for many time steps of the simulation.

DC Microgrid Bus Fuzzy Inference Stability Analysis: Estimated Domain of Attraction with Different DC Bus Capacitance

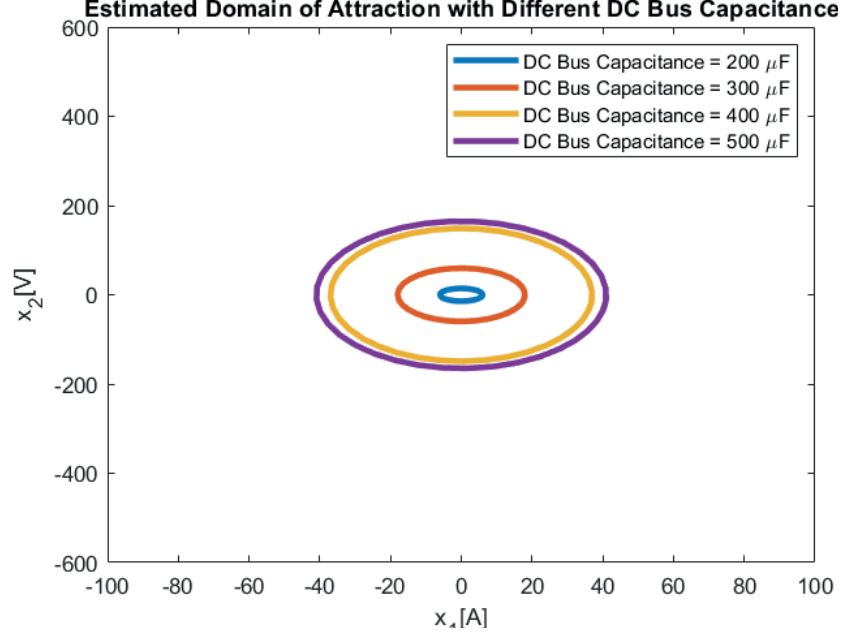

Figure 13. Domain of attraction of the $600 \mathrm{~V} / 380 \mathrm{~V}$ data center dc microgrid using varying do bus capacitance 


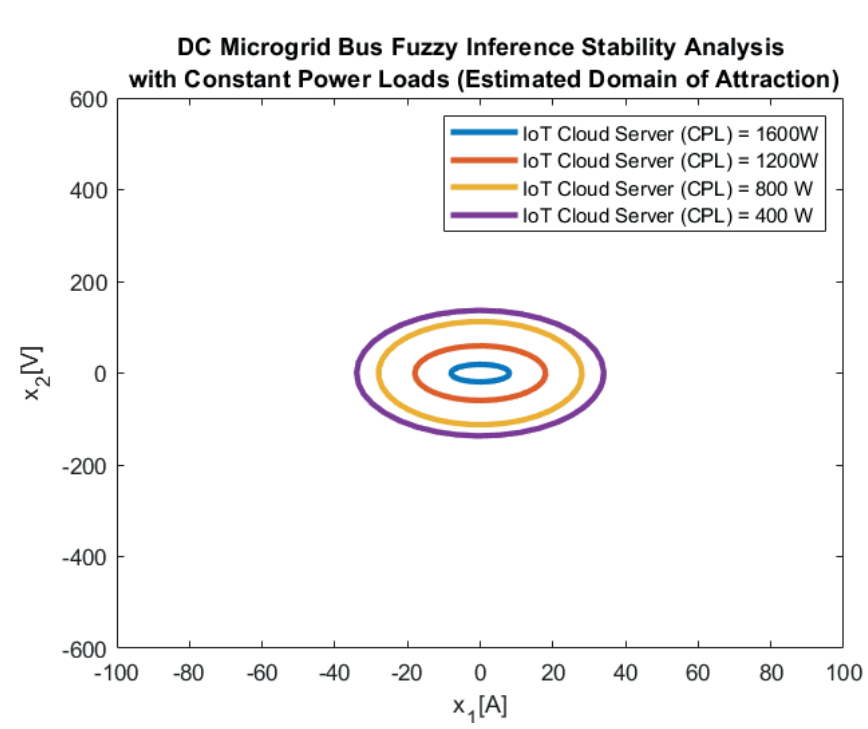

Figure 14. Domain of attraction of the $600 \mathrm{~V} / 380 \mathrm{~V}$ data center dc microgrid with varying data center server loads modeled as CPLs

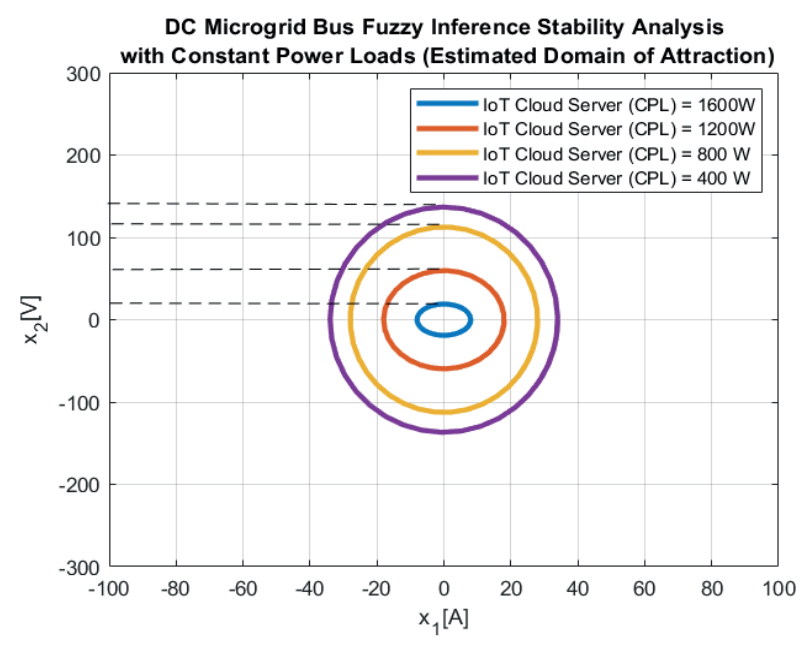

Figure 15. Domain of attraction of the $600 \mathrm{~V} / 380 \mathrm{~V}$ data center dc microgrid with varying data center server loads modeled as CPLs (enlarged voltage axis)

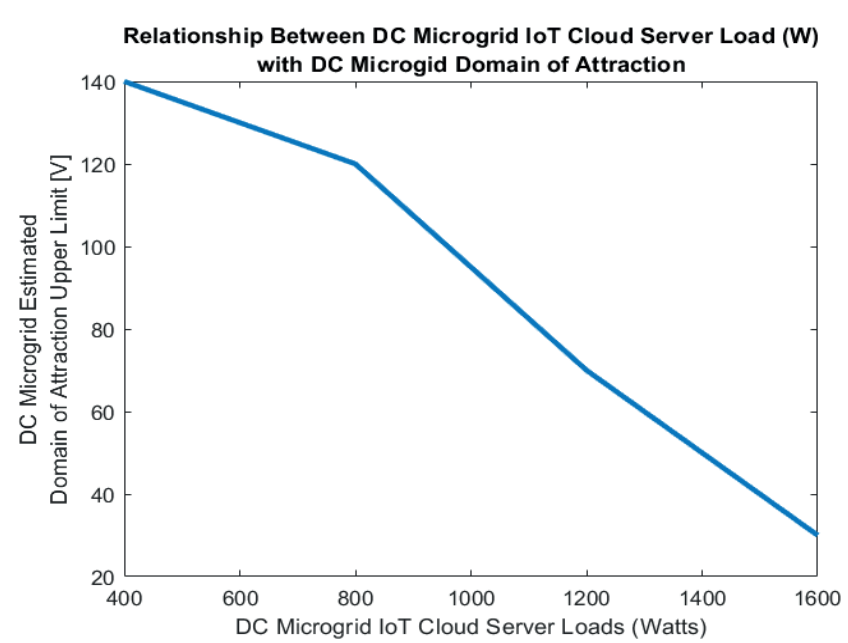

Figure 16. Comparison of the upper limit of the dc microgrid loT cloud data center server load with upper limit of the stability domain

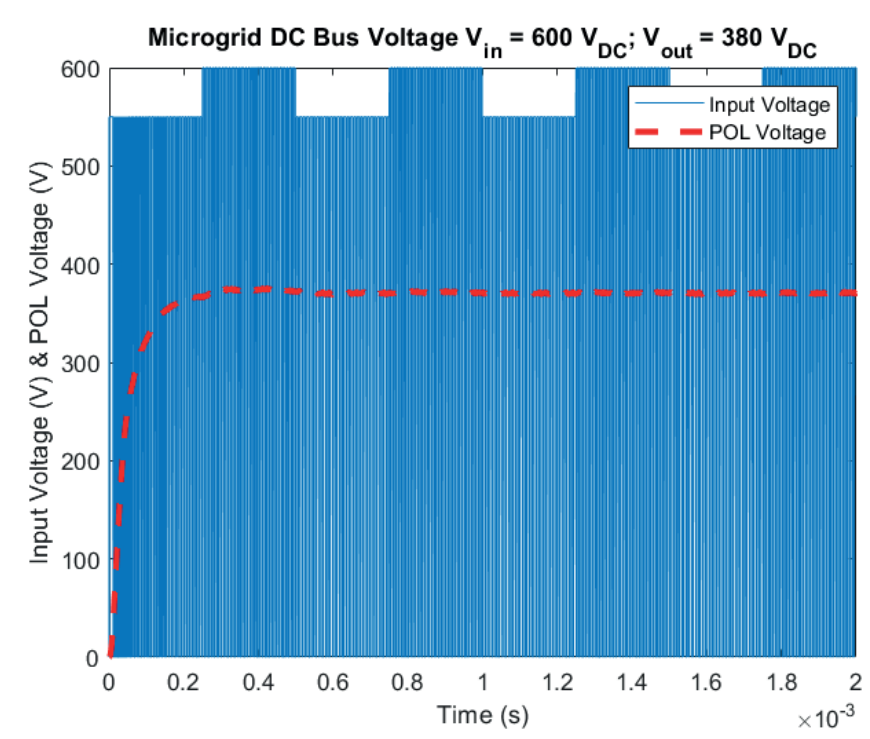

Figure 17. Existence of stable Lyapunov candidate ensures that the $600 \mathrm{~V} / 380 \mathrm{~V}$ dc microgrid is asymptotically (large signal) stable even when system input voltage experience perturbations

\section{IMPACT OF DC MICROGRID IOT SERVER CPL ON DC MICROGRID POWERLINE COMMUNICATION}

For communication purpose, the dc bus microgrid powerline can be modelled as a linear time invariant (LTI) system, and generally for LTI systems [25], [29], the group delay is a useful measure of amplitude and time distortion of signals generated by such channels [25], [29]. Suppose that $x$ is the input signal to the LTI dc bus powerline channel having impulse response $h[n]$, then the channel output $y[n]$ is,

$$
\mathrm{y}[\mathrm{n}]=\sum_{k=-\infty}^{\infty} x[k] h[n-k]
$$

which can also be represented using the discrete time Fourier transform identity as,

$$
\mathrm{Y}\left(e^{j \omega}\right)=\mathrm{H}\left(e^{j \omega}\right) \mathrm{X}\left(e^{j \omega}\right)
$$

(36) implies that the magnitude of the PLC channel and the input has multiplicative effect while their phases have additive effects [25], [29] leading to,

$$
\begin{aligned}
& \left|\mathrm{Y}\left(e^{j \omega}\right)\right|=\left|\mathrm{H}\left(e^{j \omega}\right)\right|\left|\mathrm{X}\left(e^{j \omega}\right)\right| \\
& \angle \mathrm{Y}\left(e^{j \omega}\right)=\angle \mathrm{H}\left(e^{j \omega}\right)+\angle \mathrm{X}\left(e^{j \omega}\right)
\end{aligned}
$$

In (37), $\mathrm{H}\left(\mathrm{e}^{\mathrm{i} \omega}\right)=\mathrm{H}\left(\mathrm{e}^{\mathrm{j} \omega}\right) \mid \mathrm{H}\left(0 \mid \mathrm{e}\left(\mathrm{j}^{\Phi(\omega)}\right)\right.$, and in (38), $\angle \mathrm{H}\left(\mathrm{e}^{\mathrm{j} \omega}\right)=\varnothing(\omega)=$ $\arg \left[\mathrm{H}\left(\mathrm{e}^{\mathrm{i} \omega}\right)\right]$. Thus, the phase delay $\tau_{\mathrm{p}}(\omega)$ of the LTI channel is represented

$$
\tau_{p}(\omega)=-\frac{\emptyset(\omega)}{\omega}=-\frac{\arg \left[\mathrm{H}\left(e^{j \omega}\right)\right]}{\omega}
$$

Also, the group delay $(\omega)$ of the DC powerline LTI channel can be represented as,

$$
\tau_{g}(\omega)=\operatorname{grd}\left[\mathrm{H}\left(e^{j \omega}\right)\right]=-\frac{d}{d \omega}\left\{\arg \left[\mathrm{H}\left(e^{j \omega}\right)\right]\right\}=-\frac{d}{d \omega} \varnothing(\omega)
$$


In other to evaluate the level of distortion that CPLs could generate on the dc microgrid bus powerline, the dc microgrid system of Figure 11 is simplified as shown in Figure 18 so as to represent a medium length and medium voltage dc bus powerline system [30], [31]. The inductance parameter of Figure 18, aptly represent a medium 0 length powerline such as the dc microgrid bus under consideration [30], [31], [25].

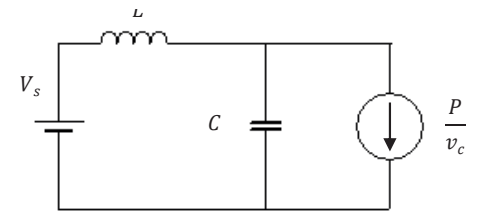

Figure 18. Simplified model of the dc microgrid powerline communication channel. A medium length, medium voltage transmission line channel is used to represent the PLC channel here.

This dc bus model in Figure 18 is a model of a second order low pass filter [32] and its transfer function can be represented [33] as

$$
H(s)=\frac{\omega^{2} o}{s^{2}+\frac{\omega_{o}}{Q} s+\omega^{2} o}
$$

By using KCL, the transfer function of the dc microgrid in (35) and Figure 18 can be represented as

$$
\frac{V_{\text {out }}}{V_{\text {in }}}=\frac{\frac{1}{L C}}{s^{2}+\mathrm{s} \frac{v_{C}}{P C}+\frac{1}{L C}}
$$

The transfer function in (42) is used to evaluate the rate of signal distortion that will be experienced by a transmitted signal when CPLs exist on the microgrid dc bus PLC channel. A $39.5 \mathrm{mH}$ inductance value [21], [23] and $500 \mu \mathrm{F}$ capacitance value is used for simulation. The in (42) is the $380 \mathrm{~V}$ dc bus voltage. $P$ is the loT data center load modeled as CPL for the dc bus microgrid. CPL power $(P)$ ratings used to represent loT server loads on the dc bus channel are $400 \mathrm{~W}, 800 \mathrm{~W}, 1000 \mathrm{~W}, 1200 \mathrm{~W}$ and $1600 \mathrm{~W}$ respectively. The rate of a baseband sine wave signal distortion as a function of delay samples using the dc bus channel parameters discussed is evaluated using Matlab and results are shown in Figure 19 to Figure 24

As shown in Figure 19 to Figure 24, the width of the filter passband is decreasing as more CPLs exist on the dc bus PLC channel. This signifies that lesser samples of the baseband channel signal will experience equal amount of distortion as more CPLs exist on the microgrid dc bus channel. As dc bus channel CPL increases, increasing samples of any signal transmitted on the dc bus channel will experience different amount of delay, leading to more signal samples arriving distorted at the receiving end.

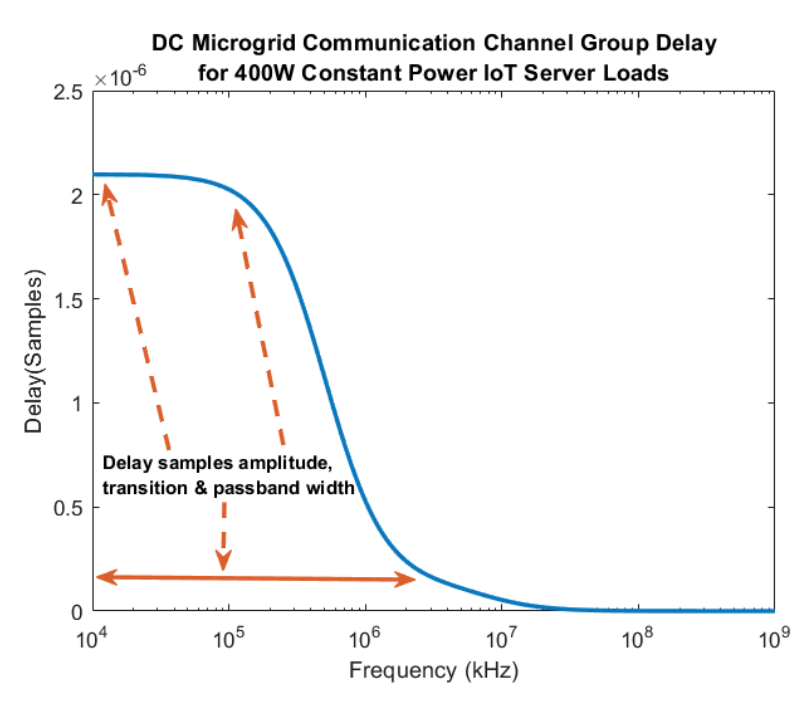

Figure 19 Group delay distortion caused by 400 W loT server CPL load on dc bus microgrid channel

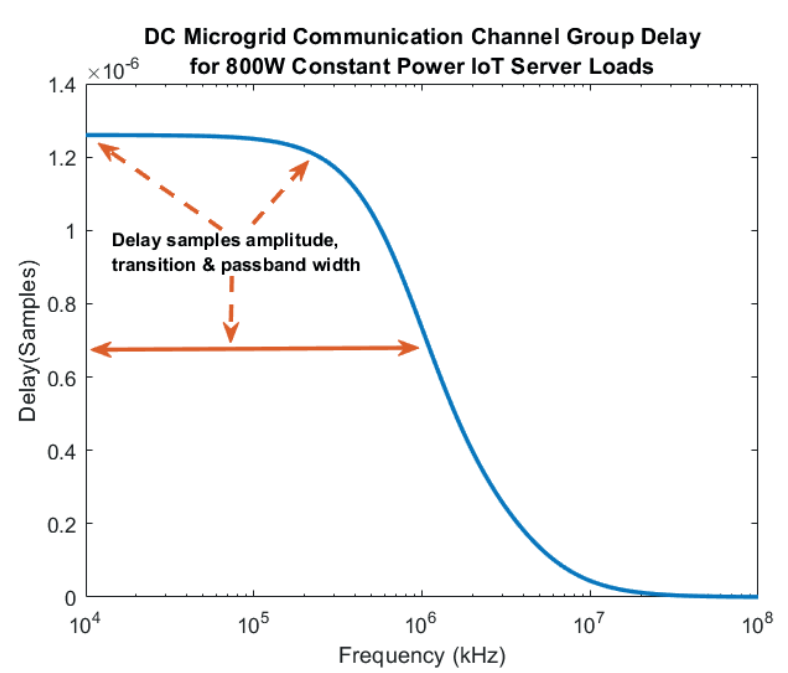

Figure 20 Group delay distortion caused by 800 W loT server CPL load on dc bus microgrid channe

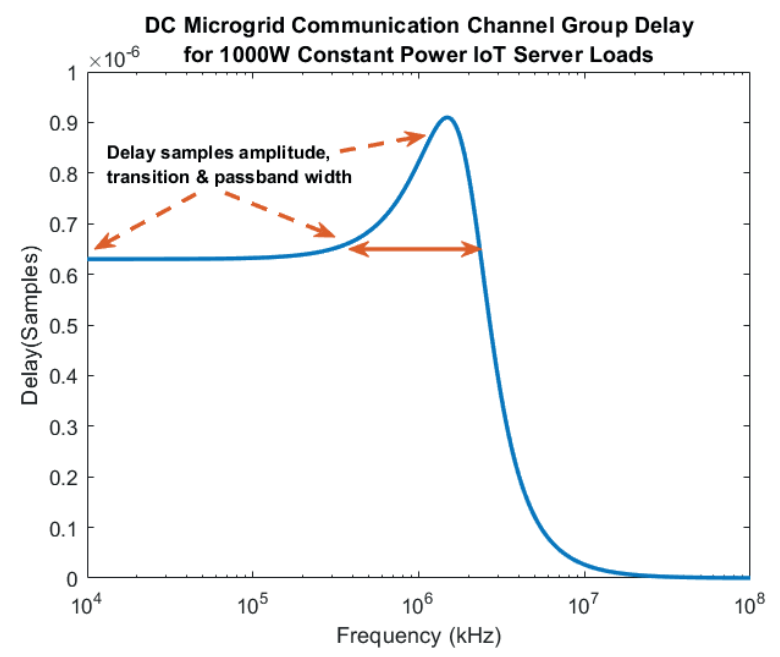

Figure 21 Group delay distortion caused by $1000 \mathrm{~W}$ loT server CPL load on dc bus microgrid channel 


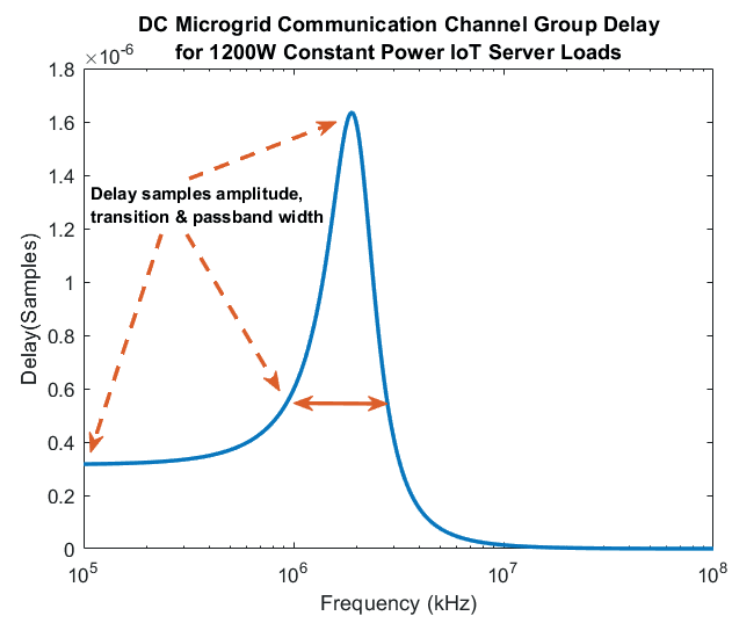

Figure 22 Group delay distortion caused by 1200 W loT server CPL load on dc bus microgrid channel

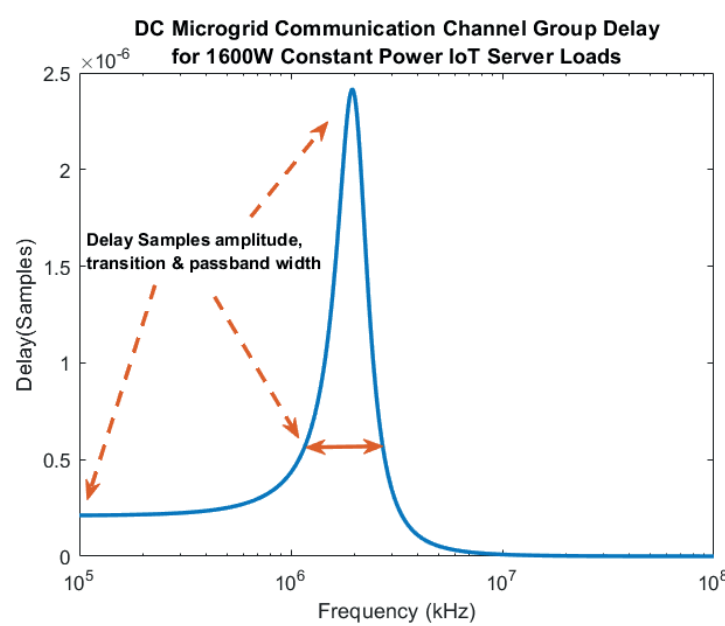

Figure 23 Group delay distortion caused by 1600 W loT server CPL load on dc bus microgrid channel

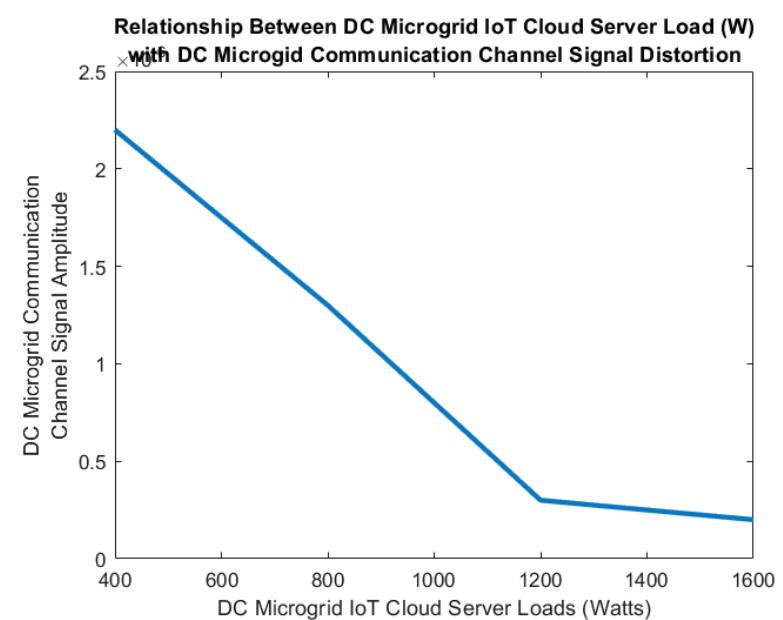

Figure 24 Baseband signal amplitude decreases as microgrid dc bus CPL loads increases.

It can also be deduced using Figure 24 that the amplitude of the baseband signal decreases progressively as more CPL exist on the dc bus channel, leading to more transmitted signal distortion. Also, as more CPLs exist on the dc bus channel, there is increasing abruptness from the filter's passband to stopband. The more abrupt a transition from the passband to stopband, the greater the delay distortion that a transmitted signal will experience across the transition band [34].

\section{CONCLUSION}

In this paper, a $600 \mathrm{~V} / 380 \mathrm{~V}$ DC-DC microgrid that can be used to represent state of the art loT data center microgrid is modeled using Matlab. Takagi-Sugeno fuzzy inference model is used to establish the domain of attraction of the dc microgrid. It is discovered that the domain of attraction, which represent the stability region of the data center microgrid increases when the dc bus channel capacitance increases. This signifies enhanced system stability. The domain of attraction also decreases with increasing CPL loT server loads on the dc bus channel. This signifies reducing system stability. PLC is being considered as a future means of monitoring the dc bus microgrid such that the power usage efficiency and stability of the data center microgrid can be improved. In this paper impact of varying CPLs such as data center loT cloud servers on signals transmitted on the dc microgrid bus PLC channel is evaluated. Our contribution in this paper, to the best of our knowledge is the first reported evaluation of the stability and measurement of the PLC channel signal distortion of the $380 \mathrm{~V} \mathrm{dc}$ microgrid when CPLs on the dc bus is varying. It is discovered that more CPLs on the PLC channel will lead to increased signal distortion on the dc bus channel when PLC is used as means of communication on the channel. Hence, to be able to use the powerline of the dc microgrid as a means of monitoring the dc microgrid stability and energy utilization, effects of varying CPLs on PLC channel must be considered. Future work in this area will include research work on delay equalizers that can be used to reduce signal distortion rate when the dc microgrid powerline is used as a means of communication, monitoring stability and energy utilization on the microgrid dc bus channel. 
REFERENCE

[1] A. Wierman, Z. Liu, I. Liu, H. Mohsenan-Rad, "Opportunity and Challenges for Data Center Demand Response", International Green Computing Conference Dallas, TX, 2014, pp. 1-10.

[2] A. Fernandez, P. Dave, "Powerline Communication in Energy Markets", White Paper, Cypress, 2011.

[3] X. Yao, "Study on DC Arc Faults in RingBus DC Microgrids with Constant Powe Loads", 2016 IEEE Energy Conversion Congress and Exposition (ECCE), Milwaukee, Wl, 2016, pp. 1-5.

[4] D. Hong, J. Lee, J. Choi, "Power Quality Monitoring System Using Power Lin Communication," IEEE, 2005.

[5] F. Yang, W. Ding, J. Song, "Non-Intrusive Power Line Quality Monitoring based on Power Line Communications," IEEE 17th Int'l Symposium on Power Line Comm. \& Its Applications, 2013.

[6] V. Dani et'al, "Power Line Carrier Communication Based on Low-cost Power Monitoring and Management System," IEEE 1st Int'। Conf. On Sustainable Green Building and Communities, December, 2016.

[7] L. Lu, G. Li, Y. Q. Song, "Power Line Carrie Communication System for Monitoring and Supervision of Feeder Equipment for MV Substation Automation," IEEE Symposium on Industrial Embedded Systems; October, 2006

[8] C. Sridhathan, and F. Samsuri, "ECG and EEG Monitoring Using Power Line Communications," ACEEE Int'l Journa on Communication, Vol. 5, No. 1, March, 2014

[9] S. Whaite, B. Grainger, and A. Kwasinski "Power Quality in DC Power Distribution "Power Quality in DC Power Distribution Systems and Microgrids," in Journal of Available:www.mdpi.com/journal/energie.

[10] P. Karlsson, J. Svensson, "DC Bus Voltage Control for a Distributed Power System", IEEE Trans. on Power Electronics, Vol. 18, No. 6, Nov. 2003.

[11] W. Tang and R. H. Lassetter, "An LVDC Industrial Power Distribution System Without Central Control Unit", in Proc. IEEEPESC Conf., Vol. 2, Galway, Ireland, June 18-23, 2000, pp. 979-984.

[12] J. LeSage, R. Longoria, W. Shutt, "Power System Stability Analysis of Synthesized Complex Impedance Loads on an Electric Ship", IEEE 2011.
[13] M. Cupelli, L. Zhu, A. Monti, "Why Idea Constant Power Loads Are Not the Wors Case Condition From a Control Standpoint", IEEE Trans. on Smart Grid, Vol. 6, No. 6, Nov. 2015.

[14] S. Augustine, M. Mishra, N. Lakshminarasamma, "Adaptive Droop Control Strategy for Load Sharing and Circulating Current Minimization in Low-Voltage Stand Alone MC Microgrid", IEEE Trans. on Sustainable DC Microgrid", IEEE Trans. on Sustaingt

[15] R. D. Middlebrook, "Design Techniques for Preventing Input Oscillations in SwitchedMode Regulators", Proceedings of Power Conf. 5, pp. A3.1-A3.16, May 1978.

[16] R. W. Erickson, "Optimal Single Resisto Damping of Input Filters", Applied Powe Electronics conf. and Exposition, APEC, 1999, pp. 1073-1079.

[17] M. Sclocchi, "Input Filter Design for Switching Power Supplies", National Semiconductor Corporation, 2010.

[18] M. M Medina, L. Qi, and K. L. Butler-Purry "A Three Phase Load Flow Algorithm for Shipboard Power Systems (SPS)", 2003 IEEE PES Transmission and Distribution Conference and Exposition, Dallas, TX, USA, pp. 227-233.

[19] M. Kabalan, P. Singh, D. Niebur, "Large Signal Lyapunov-Based Stability Studies in Microgrids: A Review", IEEE Trans. on Smart Grids; IEEE 2016.

[20] A. Emadi, A. Khaligh, C. H. Rivetta, G A. Williamson, "Constant Power Load and Negative Impedance Instability in Automotive Systems: Definition, Modeling, Stability, and Control of Powe Electronic Converters and Motor Drives", IEEE Transaction on Vehicula Technology, Vol. 55, No. 4, July 2006.

[21] D. Marx, S. Pierfederici, B. Nahid-Mobarakeh, B. Davat, "Contribution to Determination of Domain of Attraction in Powe Systems: Application to Drives With Input Filter", 2009 IEEE Industry Application Society Annual Meeting, Houston, TX, 2009, pp. 1-8.

[22] L. Herrera, “ Stability Analysis and Controller Design of DC Microgrids with Constant Power Loads", IEEE, 2015

[23] D. Marx, P. Magne, B. Nahid-Mobarakeh, S. Perfederici, and B. Davat, "Large Signal Stability Tools in DC Power Systems With Constant Power Loads and Variable Power Loads - A Review", in IEEE Tran- saction on Power Electronics, vol. 27, no. 4, pp. 1773-1787.

[24] Y-C. Chang, H-C. Chang, and C-Y. Huang, "Design and Implementation of the Battery Energy Storage System in DC Micro-Grid Systems", Journal of Energies, MDPI, June 2018.

[25] E. Oyekanlu, P. Oladele, "Smart Grid Communication over DC Powerline: Evaluation of Powerline Communication OFDM PAPR for New Types of Destabilizing Electrical Loads", IEEE/CIGRE Smart Grid Metrology Conference, Croatia, March 2018.

[26] S. H. Zak, G. Zhai, "Introduction to Linear Matrix Inequalities (LMIs)", ECE 680, Purdue University, online, available: https://engineering.purdue.edu/ECE680; October 2017

[27] P. Magne, D. Marx, B. Nahid-Mobarakeh and S. Pierfederici, "Large Signal Stabilization of a DC-Link Supplying Constan Power Load Using a Virtual capacitor Impact on Domain of Attraction", in IEEE Transaction on Industry Application, vol. 38, no. 3, pp. 878-887, June 2012

[28] M. Kabalan, P. Singh, and D. Niebur, "A Design and Optimization Tool for Inverter-Based Microgrids Using Large-Signal Nonlinear Analysis", in IEEE Transaction on Smart Grid, 2018.

[29] F. J. Carnete, L. Diaz, J. A. Cortez, J. Sanchez-Martinez, and L. M. Torres, "Time Varying Channel Emulator for Indoo Power-Line Communication", IEEE, 2008.

[30] G. Tcheslavski, "Transmission Lines", Fundamental of Power Engineering, Lamar University, 2008.

[31] Missouri S\&T University, "Voltage Regulation of a Transmission Line", online, available, www.ece.mst.edu; September, 2017.

[32] lowa Hills Software, "What is Group Delay", online, available, http://iowahills.com/ B1GroupDelay.html; September, 2017.

[33] P. Allen, "Continuous Time Filters", online, available, hasler.ece.gatech.edu/Courses/ ECE6414/Unit1/Discrete_03.pdf

[34] J. Smith, "Introduction to Digital Filters with Audio Applications", W3K Publishing, http://books.w3k.org/, 2007. 